\title{
Adverse Events and Clinic Visits following a Single Dose of Oral Azithromycin among Preschool Children: A Randomized Placebo-Controlled Trial
}

\author{
Ali Sié, ${ }^{1}$ Clarisse Dah, ${ }^{1}$ Mamadou Bountogo, ${ }^{1}$ Mamadou Ouattara, ${ }^{1}$ Eric Nebie, ${ }^{1}$ Boubacar Coulibaly, ${ }^{1}$ Jessica M. Brogdon, ${ }^{2}$ \\ William W. Godwin, ${ }^{2}$ Elodie Lebas, ${ }^{2}$ Thuy Doan, ${ }^{2,3}$ Benjamin F. Arnold, ${ }^{2,3}$ Travis C. Porco, ${ }^{2,3,4}$ Thomas M. Lietman, ${ }^{2,3,4}$ and \\ Catherine E. Oldenburg ${ }^{2,3,4 *}$ for the GAMIN Study Group \\ ${ }^{1}$ Centre de Recherche en Santé de Nouna, Nouna, Burkina Faso; ${ }^{2}$ Francis I Proctor Foundation, University of California, San Francisco, California; \\ ${ }^{3}$ Department of Ophthalmology, University of California, San Francisco, California; ${ }^{4}$ Department of Epidemiology and Biostatistics, University of \\ California, San Francisco, California
}

\begin{abstract}
Biannual mass azithromycin distribution reduces all-cause child mortality in some settings in sub-Saharan Africa; however, adverse events and short-term infectious outcomes following treatment have not been well characterized. Children aged 0-59 months were recruited in Nouna Town, Burkina Faso, and randomized 1:1 to a single directly observed oral $20 \mathrm{mg} / \mathrm{kg}$ dose of azithromycin or placebo. At 14 days after treatment, caregivers were interviewed about adverse event symptoms their child experienced since treatment and if they had sought health care for their child. All children had tympanic temperature measured at the 14-day visit. We compared adverse events and clinic visits using logistic regression models between azithromycin- and placebo-controlled children. Of 450 children enrolled, 230 were randomized to azithromycin and 220 to placebo. On average, children were aged 28 months, and $50.9 \%$ were female. Caregivers of $20 \%$ of children reported that their child experienced at least one adverse event, with no significant difference between study arms (19.9\% azithromycin; $20.0 \%$ placebo, logistic regression $P=0.96$ ). Vomiting was more often reported by caregivers of azithromycin-treated children than by those of placebo-treated children $(7.2 \%$ azithromycin, $1.9 \%$ placebo, logistic regression $P=0.01$ ). There were no significant differences in other adverse events or clinic visits. Adverse events following a single oral dose of azithromycin in preschool children were rare and mild. Azithromycin administration appears safe in this population.
\end{abstract}

\section{INTRODUCTION}

Biannual mass azithromycin distribution to preschool children has been shown to decrease all-cause child mortality in some areas of sub-Saharan Africa. ${ }^{1-3}$ The mechanism behind this effect is likely due to an overall decrease in infectious burden. ${ }^{4,5}$ However, mass administration of azithromycin may have some risk of adverse events, including mild gastrointestinal events or more severe adverse events such as infantile hypertrophic pyloric stenosis (IHPS) or allergic reaction. Adverse events among young children participating in studies of azithromycin for child mortality have not been well described.

Azithromycin for trachoma control has generally been well tolerated. Most adverse events following azithromycin distribution for trachoma control are mild gastrointestinal events, such as nausea and vomiting. ${ }^{6,7}$ No increase in adverse events was observed in children aged 1-5 months in the Niger site of the Macrolides Oraux pour Réduire les Décès avec un Oeil sur la Résistance (MORDOR) study, although there was a decrease in caregiver-reported diarrhea approximately 1 month after treatment in children receiving azithromycin compared with placebo. ${ }^{8}$ This may be reflective of a decrease in clinical diarrhea in children receiving azithromycin because of a protective effect of azithromycin, which would further support the mechanistic hypothesis of an overall reduction in infection burden following azithromycin treatment. Serious adverse events may also occur following azithromycin administration. Observational studies have indicated that young infants may be at increased risk of IHPS following macrolide use, including azithromycin, particularly when administered

\footnotetext{
*Address correspondence to Catherine E. Oldenburg, Francis I Proctor Foundation, University of California, San Francisco, 490 Illinois St., Floor 2 San Francisco, CA 94158. E-mail: catherine. oldenburg@ucsf.edu
}

before 42 days of life. ${ }^{9}$ Active surveillance for nonserious adverse events was not undertaken in children aged 6 months and older in MORDOR.

The Gut and Azithromycin Mechanisms in Infants and Neonates study was designed to assess changes in the microbiome, infection, and growth in children aged 0-59 months over a 6-month period following a single oral dose of azithromycin compared with placebo. Here, we report adverse events and clinic visits during the 2-week period immediately after treatment to better understand the safety and short-term impact of azithromycin compared with placebo in young children.

\section{METHODS}

Study setting. This study took place in Nouna town, Burkina Faso. Nouna is the capital of Kossi Province in northwestern Burkina Faso and has approximately 20,000 residents. Nouna is situated within the Nouna Health and Demographic Surveillance Site, which has been operating in Nouna town and 58 surrounding communities since $1992 .^{10}$ This study was reviewed and approved by the institutional review boards at the University of California, San Francisco, and the Comité National d'Ethique pour la Recherche (National Ethics Committee of Burkina Faso) in Ouagadougou, Burkina Faso. Written informed consent was obtained from the caregiver of each study participant.

Eligibility and recruitment. Children were eligible for participation in the trial if they were aged between 8 days and 59 months, lived in Nouna town, were planning to be available for the full 6-month duration of the study, could feed orally (to ensure they could take the study medication), and had no known allergies to macrolides. Children were recruited by mobilization in the community. Mobilizers visited households with children younger than 5 years, informed caregivers about 
the study, and caregivers were instructed to bring their children to Nouna district hospital for eligibility assessment and enrollment. To facilitate sample collection for some study outcomes, all study procedures took place at Nouna district hospital.

Baseline procedures. After the eligibility assessment, caregivers completed a brief baseline questionnaire, which included questions related to the child's breastfeeding status and maternal characteristics including the mother's age and education. Anthropometric measurements, including height, weight, and mid-upper arm circumference, were taken from all children. Standing height was taken for children who could stand or recumbent length for those who could not (ShorrBoard, Weight and Measure LLC, Olney, MD). Children were weighed standing if able or in the arms of a caregiver (Seca 874 flat floor scale, Seca GMBH \& Co., Hamburg, Germany). Height-for-age, weight-for-age, and weight-for-height Zscores were calculated based on WHO 2006 standards.

Randomization. Children were randomized in a 1:1 fashion to a single dose of oral azithromycin or placebo. The randomization list was generated without stratification or blocking by the trial biostatistician in $R$ version 3.6.1 (the $R$ Foundation for Statistical Computing, Vienna, Austria) using a masked seed value. ${ }^{11}$

Interventions and masking. Both azithromycin and placebo were prepared as a pediatric oral suspension. Weightbased dosing was used for children younger than 12 months (equivalent to $20 \mathrm{mg} / \mathrm{kg}$ ), and a height stick approximation was used for children older than 12 months, as in trachoma studies and in the MORDOR study. ${ }^{1,12}$ Azithromycin and placebo were identical in appearance and taste to facilitate masking. To ensure masking, each child's study identification number was linked to a treatment letter. Study treatment bottles were labeled with letters, and each child was treated with medication from a bottle that corresponded to their treatment letter. The only difference in the appearance of the medication bottles was the study treatment letter. Study participants, outcome assessor, and study staff and investigators were masked to treatment assignment. Treatment was administered at the Nouna district hospital following completion of all baseline assessments and was directly observed. Allocation concealment was achieved by linking study identification numbers to treatment letters that only appeared in the study's electronic data capture system after the child was enrolled.

Outcome assessment. Children returned to the hospital approximately 2 weeks after treatment. During this visit, caregivers completed a brief interview about symptoms their child experienced in the previous 2 weeks following treatment, including abdominal pain, vomiting, diarrhea, constipation, and skin rash. Caregivers were asked about experience of any of these symptoms since treatment. These adverse events were based on previous findings from azithromycin studies for child mortality and trachoma. ${ }^{6-8}$ Caregivers were also asked if they had sought care for their child in the previous 2 weeks, and if so, the diagnosis (e.g., malaria, pneumonia, and diarrhea). Each child's tympanic temperature was measured. Although no children younger than 28 days were enrolled, the study's protocol specified increased adverse event screening for these children, following an identical protocol to that of an ongoing randomized controlled trial of neonatal azithromycin distribution for the prevention of infant mortality. ${ }^{13}$ Children enrolled at $<28$ days of age are followed weekly for 3 weeks following enrollment. If a caregiver reports vomiting, children are screened for projectile vomiting and other signs of IHPS and referred for a diagnostic ultrasound if indicated.

Sample size determination. The sample size was based on the primary outcome for the trial, Shannon's diversity index of the gut microbiome.

Statistical methods. Baseline characteristics of the study sample were calculated as means and SDs for continuous variables and proportions for categorical variables. All outcomes were dichotomous in this analysis. We calculated the proportion of participants experiencing any adverse event (caregiver reported at least one) by study arm, and each adverse event individually. We calculated proportions with fever at the time of the follow-up visit (defined as tympanic temperature $>37.5^{\circ} \mathrm{C}$ ). Finally, we calculated the proportion of participants with any clinic visit and specific reasons for clinic visits. We calculated odds ratios for each outcome separately using a logistic regression model predicting the odds of the outcome with the randomized treatment arm as the predictor. We then calculated age-stratified proportions and odds ratios for all adverse events in categories of 0-11 months, 1223 months, and 24-59 months. Because the primary purpose was to evaluate safety endpoints, we did not use multiple comparison corrections to be conservative. All analyses were intention-to-treat and included all randomized children, regardless of whether or not they received their randomized assignment. All analyses were conducted in $\mathrm{R}$ version 3.6.1 (the R Foundation for Statistical Computing).

\section{RESULTS}

Of 450 children screened, all were eligible and enrolled in November 2019; 230 were randomized to azithromycin and 220 to placebo (Figure 1). No children younger than 42 days were enrolled in this study. Children were similarly aged in the azithromycin (mean age 28 months, SD 15 months) and placebo (mean age 28 months, SD 14 months) and 53\% and $49 \%$ of children in the azithromycin and placebo groups were female, respectively (Table 1). Of the 450 children enrolled, 441 $(98 \%)$ received study treatment. Of the nine children recorded to not have received study treatment, six $(66.7 \%)$ were reported to have spit up and three (33.3\%) were reported to have vomited. There were no significant differences in receiving treatment between arms (98.7\% received treatment in the azithromycin arm and $97.3 \%$ received treatment in the placebo arm, $P=0.33$ ).

Caregivers of $20 \%$ of children reported that their child had experienced at least one adverse event in the 14-day period following treatment, with no difference in report of adverse events between study arms (19.9\% azithromycin; $20.0 \%$ placebo, odds ratio [OR]: $0.99,95 \% \mathrm{Cl}: 0.62-1.59, P=0.96$; Table 2). The most commonly reported adverse event was fever, which was reported in $16.7 \%$ of children in both study arms (OR: $1.00,95 \% \mathrm{Cl}: 0.50-1.66, P>0.99$ ), followed by diarrhea (8.1\% azithromycin, $6.2 \%$ placebo, OR: $1.34,95 \%$ $\mathrm{Cl}$ : 0.04-24.0, $P=0.44)$. Vomiting was more often reported by caregivers of azithromycin-treated children than by those of placebo-treated children (7.2\% azithromycin, $1.9 \%$ placebo, OR: $4.0,95 \% \mathrm{Cl}: 1.44-14.1, P=0.01)$. There was no significant evidence of a difference in other adverse events or in tympanic temperature (Table 2). There were no differences in adverse events in age-stratified models (Table 3).

Approximately $5 \%$ of caregivers reported that they sought health care for their child during the 14-day period following 


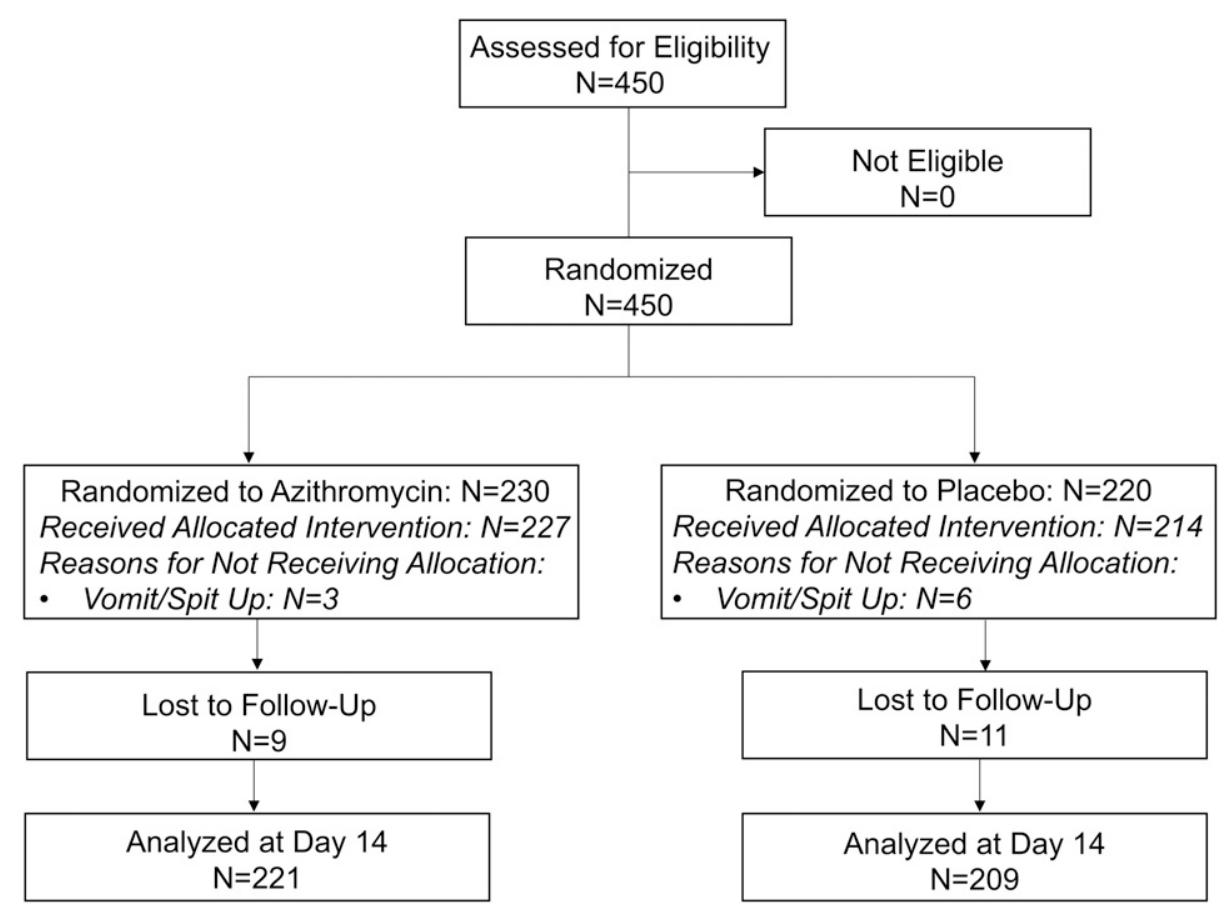

FIGURE 1. CONSORT flow diagram.

treatment. Although the occurrence of primary healthcare visits was lower in azithromycin- than in placebo-treated children, this difference was not statistically significant $(4.1 \%$ azithromycin, $6.2 \%$ placebo, OR: $0.64,95 \% \mathrm{Cl}: 0.26-1.52, P=$ 0.32; Table 4). Malaria was the most common reason for seeking care, but there was no significant difference in specific reasons for care-seeking by study arm.

\section{DISCUSSION}

We documented an increase in vomiting in the 2-week period following treatment in azithromycin- compared with placebo-treated children, consistent with well-described gastrointestinal side effects associated with azithromycin use. ${ }^{14,15}$ Biannual mass azithromycin distribution has been shown to lead to a decrease in all-cause child mortality in some settings. ${ }^{1,2,16,17}$ However, the MORDOR study was a large simple trial, ${ }^{18}$ and was not designed to evaluate individual-level outcomes, including adverse events. A substudy of the original MORDOR study demonstrated no significant difference in adverse events in children aged 1-5 months. ${ }^{8}$ In this sub-study, there was no significant difference in vomiting in azithromycin- compared with placebotreated infants. However, vomiting was much more common in both arms in the infant sub-study than in the present study, as expected with this younger age-group. Any increase in vomiting in azithromycin-treated infants may have been masked by an overall increase in vomiting between the two arms. Studies of azithromycin administration to neonates for a variety of indications have generally found low prevalence of gastrointestinal side effects, with the most common side effect reported being vomiting. ${ }^{14,15}$ Vomiting can be a symptom of IHPS, a rare but serious condition in neonates and young infants that requires surgical intervention. In observational studies, macrolide use has been suggested to increase the risk of IHPS in infants younger than 42 days. ${ }^{9}$ Although we did not enroll any infants younger than 42 days and this study was not specifically designed to evaluate the role of macrolides on IHPS, we had rigorous screening protocols in place for children in this age range. No deaths occurred during the present

TABLE 1

Baseline characteristics by study arm $(N=450)$

\begin{tabular}{lrr}
\hline & Azithromycin $(N=230)$ & Placebo $(N=220)^{\star}$ \\
\hline Female gender, $n(\%)$ & $122(53.0)$ & $107(48.9)$ \\
Mean age (SD) (months) & $28(15)$ & $28(14)$ \\
Currently breastfeeding, $n$ (\%) & $93(40.4)$ & $78(35.6)$ \\
Mean weight-for-height Z-score (SD) & $-0.54(1.3)$ & $-0.35(1.2)$ \\
Mean height-for-age Z-score (SD) & $-1.0(1.3)$ & $-0.9(1.4)$ \\
Mean mid-upper arm circumference (SD) & $14.2(1.3)$ & $14.4(1.2)$ \\
$\quad$ (cm) & $28(6.5)$ & $28(6.4)$ \\
Mean mother's age (SD) (years) & $121(55.0)$ \\
Mother's education, $n$ (\%) & $120(52.2)$ & $56(25.5)$ \\
None & $61(26.5)$ & $42(19.1)$ \\
Primary & $49(21.3)$ & \\
Secondary or higher & & \\
\hline
\end{tabular}

\footnotetext{
* Baseline survey missing for one participant in the placebo arm; responses may not add to $100 \%$.
} 
TABLE 2

Adverse events 14 days following azithromycin or placebo treatment among children aged 0-59 months $(N=430)$

\begin{tabular}{|c|c|c|c|c|}
\hline & Azithromycin (N=221), $n(\%)$ & Placebo $(N=209), n(\%)$ & OR (95\% Cl) & $P$-value \\
\hline Any adverse event* & $44(19.9)$ & $42(20.0)$ & $0.99(0.62-1.59)$ & 0.96 \\
\hline Fever (self-report)* $^{\star}$ & $37(16.7)$ & $35(16.7)$ & $1.00(0.60-1.66)$ & $>0.99$ \\
\hline Abdominal pain* & 0 & $2(1.0)$ & N/A & N/A \\
\hline Vomiting* & 16 (7.2) & $4(1.9)$ & $4.0(1.44-14.1)$ & 0.01 \\
\hline Diarrhea* $^{*}$ & $18(8.1)$ & $13(6.2)$ & $1.34(0.64-2.86)$ & 0.44 \\
\hline Constipation* & $1(0.4)$ & $1(0.5)$ & $0.95(0.04-24.0)$ & 0.97 \\
\hline Skin rash* & 0 & $1(0.5)$ & $\mathrm{N} / \mathrm{A}$ & $N / A$ \\
\hline Fever, tympanic $†$ & $21(9.5)$ & $14(6.7)$ & $1.45(0.72-2.99)$ & 0.30 \\
\hline
\end{tabular}

$\mathrm{N} / \mathrm{A}=$ not applicable.

${ }^{*}$ Care-giver reported adverse events occurring at any time during the 14-day posttreatment period.

† Tympanic temperature $>37.5^{\circ} \mathrm{C}$ at the time of the 14-day study visit.

study, and no children were diagnosed with IHPS, suggesting that none of the vomiting cases reported in this study were related to IHPS. An ongoing large randomized controlled trial is evaluating the safety of azithromycin specifically in neonates, and is expected to provide more definitive evidence of any effect of azithromycin on IHPS in neonates. ${ }^{13}$

We were unable to demonstrate a difference in other adverse events (e.g., diarrhea and fever) or clinic visits between children treated with azithromycin compared with placebotreated children. These adverse events could be due to common childhood infections (e.g., enteric pathogens or malaria). They could be either decreased (via reduction in infection burden) or increased (via common gastrointestinal side effects of azithromycin, in the case of diarrhea) in children receiving azithromycin compared with placebo. An analysis of a sub-study of the MORDOR trial found a significant decrease in malaria parasitemia in communities receiving azithromycin compared with placebo, ${ }^{4}$ and azithromycin for trachoma studies has found decreases in other infectious causes of mortality, including lower respiratory tract infection and diarrhea. ${ }^{19,20}$ An individually randomized study adding azithromycin to seasonal malaria chemoprevention (SMC) in Mali and Burkina Faso found a decrease in clinic visits and nonmalarial fever in children receiving azithromycin plus SMC compared with SMC alone. ${ }^{21}$ The same study showed no difference in adverse events between study arms. ${ }^{21}$ There are several reasons why our results may have differed from previous studies. First, reductions in childhood infections may be mediated by reduction in community transmission rather than individual-level effects. Both MORDOR and trachoma studies treat entire communities, and children may benefit from the effects of such mass community treatment because of reductions in pathogen transmission. Individually treated children would not benefit from an overall reduction in community transmission. Second, we assessed outcomes at 14 days after treatment. The duration may have been too short to show meaningful effects, and any effects likely would be via treatment of existing infection. The study was designed and powered for the microbiome outcome, which is more sensitive than the binary indicators in this analysis, and if the effect of treatment were via reduction in current infection, the study may have been underpowered to detect effects.

The results of this study must be considered in the context of several limitations. Children in this study were recruited in Nouna

TABLE 3

Age-stratified adverse events following azithromycin or placebo treatment among children aged 0-59 months $(N=430)$

\begin{tabular}{|c|c|c|c|c|}
\hline & Azithromycin $N=23, n(\%)$ & Placebo $N=26, n(\%)$ & OR $(95 \% \mathrm{Cl})$ & $P$-value \\
\hline \multicolumn{5}{|l|}{ Age $0-11$ months ${ }^{\star}$} \\
\hline Any adverse event $†$ & $9(39.1)$ & $7(26.9)$ & $1.74(0.53-6.0)$ & 0.37 \\
\hline Fever (self-report)† & $8(34.8)$ & $6(23.1)$ & $1.78(0.51-6.47)$ & 0.37 \\
\hline Abdominal paint & 0 & 0 & N/A & N/A \\
\hline Vomiting† & $4(17.4)$ & $1(3.9)$ & $5.26(0.71-107.5)$ & 0.15 \\
\hline Diarrhea† & $3(13.0)$ & $1(3.9)$ & 3.75 (0.44 to 79.1$)$ & 0.27 \\
\hline Constipation† & $1(4.4)$ & 0 & N/A & N/A \\
\hline Skin rash† & 0 & 0 & N/A & N/A \\
\hline Age $12-23$ months & $N=75$ & $N=52$ & & \\
\hline Any adverse event $†$ & $15(20.0)$ & $13(25.0)$ & $0.75(0.32-1.76)$ & 0.50 \\
\hline Fever (self-report) $\dagger$ & $12(16.0)$ & $9(17.3)$ & $0.91(0.35-2.41)$ & 0.85 \\
\hline Abdominal pain $†$ & 0 & $1(1.9)$ & N/A & $\mathrm{N} / \mathrm{A}$ \\
\hline Vomitingt & $5(6.7)$ & 0 & N/A & N/A \\
\hline Diarrhea† & $11(14.7)$ & $8(15.4)$ & $0.95(0.35-2.62)$ & 0.91 \\
\hline Constipation $†$ & 0 & 0 & N/A & N/A \\
\hline Skin rash† & 0 & 0 & $\mathrm{~N} / \mathrm{A}$ & N/A \\
\hline Age $24-59$ months & $N=123$ & $N=131$ & & \\
\hline Any adverse event $\dagger$ & $20(16.3)$ & $22(16.8)$ & $0.96(0.49-1.87)$ & 0.91 \\
\hline Fever (self-report) $t$ & $17(13.8)$ & $20(15.3)$ & $0.89(0.44-1.79)$ & 0.74 \\
\hline Abdominal pain $†$ & 0 & $1(0.8)$ & N/A & N/A \\
\hline Vomiting & $7(5.7)$ & $3(2.3)$ & $2.57(0.70-12.2)$ & 0.18 \\
\hline Diarrhea† & $4(3.3)$ & $4(3.1)$ & $1.07(0.25-4.60)$ & 0.93 \\
\hline Constipation $\dagger$ & 0 & $1(0.8)$ & N/A & N/A \\
\hline Skin rash† & 0 & $1(0.8)$ & $\mathrm{N} / \mathrm{A}$ & N/A \\
\hline
\end{tabular}

(ing

$\dagger$ Care-giver reported adverse events occurring at any time during the 14-day post-treatment period. 
TABLE 4

Clinic visits 14 days following azithromycin or placebo treatment among children aged 0-59 months $(N=430)$

\begin{tabular}{|c|c|c|c|c|}
\hline & Azithromycin $(N=221), n(\%)$ & Placebo $(N=209), n(\%)$ & OR $(95 \% \mathrm{Cl})$ & $P$-value \\
\hline Any clinic visit & $9(4.1)$ & $13(6.2)$ & 0.64 (0.26 to 1.52$)$ & 0.32 \\
\hline Reason: malaria & $6(2.7)$ & $5(2.4)$ & 1.14 (0.34 to 4.01$)$ & 0.83 \\
\hline Reason: diarrhea & $1(0.4)$ & $1(0.5)$ & 0.95 (0.04 to 24.0$)$ & 0.97 \\
\hline Reason: pneumonia & 0 & $1(0.5)$ & $\mathrm{N} / \mathrm{A}$ & $\mathrm{N} / \mathrm{A}$ \\
\hline
\end{tabular}

town, which is peri-urban and may have better access to health care than rural areas, higher vaccination coverage, and lower mortality rates. Although we expect adverse events following azithromycin to be broadly similar in children in rural versus urban settings, the prevalence of infectious outcomes may have been lower in these children than would be expected in a more rural setting. As previously mentioned, this study was powered for the primary outcome (microbiome) and was likely underpowered to detect differences in less common outcomes, such as clinic visits. With the exception of tympanic temperature, outcomes were reported by caregivers who may have over- or underreported outcomes. However, because the study was placebocontrolled and masked, we do not believe reporting would have been differential with respect to study arm, and thus would attenuate effects toward the null. Furthermore, the short duration of follow-up would likely reduce the probability of misclassification of outcomes by caregiver report.

Single oral dose of azithromycin led to an increase in vomiting over the 2-week period following treatment, but no other differences in adverse events posttreatment. Most adverse events following azithromycin are mild and gastrointestinal, so this result was not surprising. These data support the safety of azithromycin when given to young children for prevention of mortality.

Received August 11, 2020. Accepted for publication October 16, 2020.

Published online December 21, 2020.

Note: Supplemental file appears at www.ajtmh.org.

Financial support: The GAMIN trial was supported by the Bill \& Melinda Gates Foundation (OPP1187628). Azithromycin and matching placebo were donated by Pfizer, Inc (New York, NY). T. D. was supported in part by a Research to Prevent Blindness Career Development Award.

Authors' addresses: Ali Sié, Clarisse Dah, Mamadou Bountogo, Mamadou Ouattara, Eric Nebie, and Boubacar Coulibaly, Centre de Recherche en Santé de Nouna, Burkina Faso, E-mails: sieali@yahoo.fr, n.clarissedah@yahoo.fr, drbountogo@yahoo.fr, md.ouattara@yahoo.fr, neric83@yahoo.fr, boubacar@fasonet.bf. Jessica M. Brogdon, William W. Godwin, Elodie Lebas, Thuy Doan, Benjamin F. Arnold, Travis C. Porco, Thomas M. Lietman, and Catherine E. Oldenburg, Francis I Proctor Foundation, University of California, San Francisco, CA, E-mails: jessica.brogdon@ucsf.edu, william.godwin@ucsf.edu, elodie.lebas@ ucsf.edu, thuy.doan@ucsf.edu, ben.arnold@ucsf.edu, travis.porco@ ucsf.edu, tom.lietman@ucsf.edu, and catherine.oldenburg@ucsf.edu.

This is an open-access article distributed under the terms of the Creative Commons Attribution (CC-BY) License, which permits unrestricted use, distribution, and reproduction in any medium, provided the original author and source are credited.

\section{REFERENCES}

1. Keenan JD et al., 2018. Azithromycin to reduce childhood mortality in sub-Saharan Africa. N Engl J Med 378: 1583-1592.

2. Keenan JD et al., 2019. Longer-term assessment of azithromycin for reducing childhood mortality in Africa. N Engl $J$ Med 380: 2207-2214.
3. Oldenburg CE et al., 2019. Mass azithromycin distribution to prevent childhood mortality: a pooled analysis of cluster randomized trials. Am J Trop Med Hyg 100: 691-695.

4. Arzika AM et al., 2019. Biannual mass azithromycin distributions and malaria parasitemia in pre-school children in Niger: a clusterrandomized, placebo- controlled trial. PLoS Med 16: e1002835.

5. Keenan JD et al.,2020. Cause-specific mortality of children younger than 5 years in communities receiving biannual mass azithromycin treatment in Niger: verbal autopsy results from a cluster-randomised controlled trial. Lancet Glob Heal 8: 288-295.

6. Ayele B, Gebre T, House JI, Zhou Z, McCulloch CE, Porco TC, Gaynor BD, Emerson PM, Lietman TM, Keenan JD, 2011. Adverse events after mass azithromycin treatments for trachoma in Ethiopia. Am J Trop Med Hyg 85: 291-294.

7. Astale $T$ et al., 2019. Self-reported side effects following mass administration of azithromycin to eliminate trachoma in Amhara, Ethiopia: results from a region-wide population-based survey. Am J Trop Med Hyg 100: 696-699.

8. Oldenburg CE et al., 2018. Safety of azithromycin in infants under six months of age in Niger: a community randomized trial. PLOS Negl Trop Dis 12: e0006950.

9. Eberly MD, Eide MB, Thompson JL, Nylund CM, 2015. Azithromycin in early infancy and pyloric stenosis. Pediatrics 135: 483-488.

10. Sie A, Louis VR, Gbangou A, Müller O, Niamba L, Stieglbauer G, Yé M, Kouyaté B, Sauerborn R, Becher H, 2010. The Health and demographic surveillance system (HDSS) in Nouna, Burkina Faso, 1993-2007. Glob Health Action 3: 5284.

11. Porco TC, Stoller NE, Keenan JD, Bailey RL, Lietman TM, 2015. Public key cryptography for quality assurance in randomization for clinical trials. Contemp Clin Trials 42: 167-168.

12. Munoz B, Solomon AW, Zingeser J, Barwick R, Burton M, Bailey R, Mabey D, Foster A, West SK, 2002. Antibiotic dosage in trachoma control Programs: height as a surrogate for weight in children. Invest Ophthalmol Vis Sci 44: 1464-1469.

13. Sie A et al., 2019. Neonatal azithromycin administration to prevent infant mortality: study protocol for a randomised controlled trial. BMJ Open 9: e031162.

14. Zikic A, Schünemann $H, W i T$, Lincetto $O$, Broutet $N$, Santesso $N$, 2018. Treatment of neonatal chlamydial conjunctivitis: a systematic review and meta-analysis. J Pediatr Infect Dis Soc 7: 107-115.

15. Smith C, Egunsola O, Choonara I, Kotecha S, Jacqz-Aigrain E, Sammons H, 2015. Use and safety of azithromycin in neonates: a systematic review. BMJ Open 5: e008194.

16. Oron AP, Burstein R, Mercer LD, Arzika AM, Kalua K, Mrango Z, West SK, Bailey RL, Porco TC, Lietman TM, 2019. Effect modification by baseline mortality in the MORDOR azithromycin trial. Am J Trop Med Hyg 103: 1295-1300.

17. Porco TC et al., 2019. Efficacy of mass azithromycin distribution for reducing childhood mortality across geographic regions. Am J Trop Med Hyg 103: 1291-1294.

18. Yusuf S, Collins R, Peto R, 1984. Why do we need some large, simple trials? Stat Med 3: 409-420.

19. Coles CL, Seidman JC, Levens J, Mkocha H, Munoz B, West S, 2011. Association of mass treatment with azithromycin in trachoma-endemic communities with short-term reduced risk of diarrhea in young children. Am J Trop Med Hyg 85: 691-696.

20. Fry AM, Jha HC, Lietman TM, Chaudhary JSP, Bhatta RC, Elliott J, Hyde T, Schuchat A, Gaynor B, Dowell SF, 2002. Adverse and beneficial secondary effects of mass treatment with azithromycin to eliminate blindness due to trachoma in Nepal. Clin Infect Dis 35: 395-402.

21. Chandramohan D et al., 2019. Effect of adding azithromycin to seasonal malaria chemoprevention. $N$ Engl J Med 380: 2197-2206. 